\title{
Demonstrating the AKLT spectral gap on 2D degree-3 lattices
}

\author{
Nicholas Pomata ${ }^{1}$ and Tzu-Chieh Wei ${ }^{1,2}$ \\ ${ }^{1}$ C. N. Yang Institute for Theoretical Physics and Department of Physics and Astronomy, \\ State University of New York at Stony Brook, Stony Brook, NY 11794-3840, USA \\ ${ }^{2}$ Institute for Advanced Compuational Science, State University \\ of New York at Stony Brook, Stony Brook, NY 11794-5250, USA
}

(Dated: November 11, 2019)

\begin{abstract}
We establish that the spin-3/2 AKLT model on the honeycomb lattice has a nonzero spectral gap. We use the relation between the anticommutator of two projectors and their sum, and apply it to related AKLT projectors that occupy plaquettes or other extended regions. We analytically reduce the complexity in the resulting eigenvalue problem and use a Lanczos numerical method to show that the required inequality for the nonzero spectral gap holds. This approach is also successfuly applied to several other spin-3/2 AKLT models on degree-3 semiregular tilings, such as the squareoctagon, star and cross lattices, where the complexity is low enough that exact diagonalization can be used instead of the Lanczos method. In addition, we also close the previously open cases in the singly decorated honeycomb and square lattices.
\end{abstract}

\section{INTRODUCTION}

The question of the existence of a spectral gap above the lowest energy state(s) plays a key factor in topological phases of matter and many properties of interacting systems [1-3]. It is fundamentally related to the Lieb-Schultz-Mattis theorem [4] and generalizations thereof [ [5, [6] as well as the Haldane gapped phases [7], where there is a symmetry in the system. A nonzero spectral gap often implies exponential decay of ground-state correlation functions [8]. The latter property implies the former if the system is Lorenz invariant, but this is often not the case in condensed-matter physics. Affleck, Kennedy, Lieb and Tasaki (AKLT) constructed a family of two-dimensional spin models with isotropic spinspin interaction [9], generalizing their spin-1 chain [10], now recognized as a paradigmatic example of a symmetry protected topological phase. The ground state of the $2 \mathrm{D}$ AKLT model on both the honeycomb and square lattices display exponentially decaying correlations [9, 11]. However, the gappedness of the two models was not proved rigorously for more than three decades.

Here, we close the loop by proving the existence of spectral gap in the spin-3/2 AKLT models on four 2D Archimedean degree-3 lattices: honeycomb, squareoctagon, star and cross lattices. For the original spin- $3 / 2$ AKLT model, on the honeycomb lattice, the existence of a spectral gap was independently established in a very recent work by Lemm, Sandvik, and Wang [12] using a different, also numerically-assisted, method. We also show the gappedness of the hybrid AKLT models on the decorated honeycomb and the decorated lattices, where a spin- 1 site is added to every edge of the undecorated lattice. These two cases were left open in recent studies of AKLT models on decorated lattices [13, 14]. We note that the AKLT models on the above lattices except the honeycomb were not considered in the original work, but they are as important as the honeycomb, as all of them provide an example of symmetry protected topological phases or Haldane phases, as well as utility in realizing universal quantum computations using the measurement-based approach 15 -19].

Although the existence of the gap had not been proven, numerical tensor-network methods were used to estimate its value 20 22], which was shown to be of order 0.1 for the honeycomb model. A combination of analytics and numerics was recently used to show the spectral gap in the AKLT hexagonal chain 23]. Consideration of decorated lattices has recently led to analytic proofs of gaps for 2D AKLT models on the decorated honeycomb lattice 13., and later on the decorated square and other lattices [14]. The latter two works left open the question of whether AKLT models on the singly decorated honeycomb and square lattices are also gapped, which we demonstrate postively below.

\section{CONSTRUCTING HAMILTONIAN TERMS}

As in [13] and [14], and based on Lemma 6.3 of [24], in order to establish the existence of a gap we first extend the support of individual Hamiltonian terms. To explain this in a uniform manner, we should introduce some notation. For some subgraph $\Gamma$ of the full lattice $\Lambda$,

- We let $H_{\Gamma}$ be the AKLT Hamiltonian defined on that subgraph,

$$
H_{\Gamma}=\sum_{e \in \Gamma} H^{(e)},
$$

where, for an edge $e, H^{(e)}$ projects onto the maximum spin subspace of the two vertices which $e$ joins, which corresponds to the combined coordination number $\left(z+z^{\prime}+2\right) / 2$.

- We let $\Psi_{\Gamma}$ be the AKLT construction on the subgraph $\Gamma$, obtained by taking the spin- $\frac{z}{2}$ projector on each vertex in $\Gamma$ (represented as a rank- $(z+1)$ tensor) and contracting with them the spin-singlet 
state on each edge in $\Gamma$. This leaves us with both virtual and physical indices; we group the former into a Hilbert space $\mathcal{H}_{\text {virt }}^{\Gamma}$ and the latter into a Hilbert space $\mathcal{H}_{\text {phys }}^{\Gamma}$, so that we can write $\Psi_{\Gamma}$ as a linear transformation from $\mathcal{H}_{\text {virt }}^{\Gamma}$ to $\mathcal{H}_{\text {phys }}^{\Gamma}$.

- We write the singular value decomposition $\Psi_{\Gamma}=$ $V_{\Gamma} \Sigma_{\Gamma} U_{\Gamma}^{\dagger}$, where we omit the trivial singular values, i.e. the space $\mathcal{H}_{\Gamma}$ that $\Sigma_{\Gamma}, V_{\Gamma}$, and $U_{\Gamma}$ act on is truncated such that the latter is full-rank.

- With $\mathcal{H}_{\Gamma}$ defined as such,

$$
\Pi_{\Gamma} \equiv U_{\Gamma} U_{\Gamma}^{\dagger}
$$

is the projector onto the image of $\Psi_{\Gamma}$, which ought to equal the kernel of $H_{\Gamma}$.

Then we will define new Hamiltonian terms, of the form $\tilde{H}_{i}=\mathbb{1}_{\Gamma_{i} \text {,phys }}-\Pi_{\Gamma_{i}}$, by the subgraph $\Gamma_{i}$ on which the term is supported; in order to compare this to the original AKLT Hamiltonian, we find a $\gamma_{0}$ such that

$$
\sum_{e \in \Gamma_{i}} \frac{1}{n_{e}} H^{(e)} \geq \gamma_{0} \tilde{H}_{i}
$$

where $n_{e}$ is the number of $i$ for which $e \in \Gamma_{i}$. (Note that the frustration-freeness of the AKLT Hamiltonian allows us to vary the coefficients of the terms comprising $H_{\Gamma}$ without altering the ground space thereof.) In this case,

$$
H=\sum_{e \in \Lambda} H^{(e)} \geq \gamma_{0} \sum_{i} \tilde{H}_{i} \equiv \tilde{H}
$$

bounds the original Hamiltonian $H$ with the new Hamiltonian $\tilde{H}$.

Having done this, we complete the bound by squaring the altered Hamiltonian: if, for some $\tilde{\gamma}>0$,

$$
\tilde{H}^{2} \geq \tilde{\gamma} \tilde{H}
$$

then $\tilde{H}$ has a gap $\tilde{\gamma}$.

Since the $\tilde{H}_{i}$ are projectors, we can write

$$
\begin{aligned}
\tilde{H}^{2}=\sum_{i, j} \tilde{H}_{i} \tilde{H}_{j} & =\sum_{i} \tilde{H}_{i}+\sum_{i \neq j} \tilde{H}_{i} \tilde{H}_{j} \\
& \geq \tilde{H}+\sum_{\langle i, j\rangle}\left\{\tilde{H}_{i}, \tilde{H}_{j}\right\} \\
& \geq(1-\eta z) \tilde{H},
\end{aligned}
$$

where the sum in (7) is over "nearest neighbors" $\langle i, j\rangle$ such that $\Gamma_{i} \cap \Gamma_{j} \neq \varnothing, z$ being the (maximal) number of nearest neighbors per term, and we define $\eta$ to be the maximal number such that

$$
\{E, F\} \equiv E F+F E \geq-\eta(E+F)
$$

for all $E=\tilde{H}_{i}$ and $F=\tilde{H}_{j}$ nearest neighbors in the above sense.

In [14], we determine the following properties which we can use to simplify (9):
1. The $\eta$ which is optimal in (9) for $E=\mathbb{1}-\Pi_{\Gamma_{i}}$ and $F=\mathbb{1}-\Pi_{\Gamma_{j}}$, then it is also optimal for $E=\Pi_{\Gamma_{i}}$ and $F=\Pi_{\Gamma_{j}}$.

2. For $\eta$ optimal in (9), we find that $1-\eta$ is the least noninteger eigenvalue of $E+F$; likewise $1+\eta$ is the greatest noninteger eigenvalue of $E+F$.

3. If a projector $A=U_{A} U_{A}^{\dagger}$ commutes with both $E$ and $F$, in addition to which $E A=E$, then the "reduced" operator $U_{A}^{\dagger}(E+F) U_{A}$ has the same noninteger eigenvalues as $E+F$.

The final element we need is the form of the projectors $A$. We first note that, if $\Gamma \cap \Gamma^{\prime}=\varnothing$, then, trivially, $\Pi_{\Gamma} \otimes \mathbb{1}_{\Gamma^{c}}$ commutes with $\Pi_{\Gamma^{\prime}} \otimes \mathbb{1}_{\Gamma^{\prime c}}$. More subtly,

Proposition 1 If $\Gamma^{\prime} \subset \Gamma$, then $\left(\Pi_{\Gamma^{\prime}} \otimes \mathbb{1}_{\Gamma \backslash \Gamma^{\prime}}\right) \Pi_{\Gamma}=\Pi_{\Gamma}$.

In addition to factorizing the physical space $\mathcal{H}_{\text {phys }}^{\Gamma}$ into $\mathcal{H}_{0} \equiv \mathcal{H}_{\text {phys }}^{\Gamma^{\prime}}$ and $\mathcal{H}_{1} \equiv \mathcal{H}_{\text {phys }}^{\Gamma \backslash \Gamma^{\prime}}$, we need to examine the interplay of virtual (bond) spaces. We can do so in a straightforward way, by introducing an operator $X: \mathcal{H}_{\text {virt }}^{\Gamma^{\prime}} \otimes \mathcal{H}_{\text {virt }}^{\Gamma \backslash \Gamma^{\prime}} \rightarrow \mathcal{H}_{\text {virt }}^{\Gamma}$ which corresponds to contraction with the singlet on those pairs of indices which represent the same edge, and which acts as the identity on all other indices. Then we can write the AKLT construction on $\Gamma$ as

$$
\Psi_{\Gamma}=X\left(\Psi_{\Gamma^{\prime}} \otimes \Psi_{\Gamma \backslash \Gamma^{\prime}}\right)
$$

This implies, in particular, that the image of $\Psi_{\Gamma}$ (and therefore of $\Pi_{\Gamma}$ ) is a subset of the image of $\Psi_{\Gamma^{\prime}} \otimes \mathbb{1}_{\Gamma \backslash \Gamma^{\prime}}$ (and therefore of $\Pi_{\Gamma^{\prime}} \otimes \mathbb{1}_{\Gamma \backslash \Gamma^{\prime}}$ ). The relationship between these projectors follows immediately.

We then apply three simplifying projectors to $E$ and $F$, generated from the disjoint subgraphs $L=\Gamma_{i} \backslash \Gamma_{j}$, $R=\Gamma_{j} \backslash \Gamma_{i}$, and $C=\Gamma_{i} \cap \Gamma_{j}$. That is, we use

$$
\begin{aligned}
U_{E}^{\prime} & \equiv\left(U_{L}^{\dagger} \otimes U_{C}^{\dagger}\right) U_{E}: \mathcal{H}_{L} \otimes \mathcal{H}_{C} \rightarrow \mathcal{H}_{E} \\
U_{F}^{\prime} & \equiv\left(U_{R}^{\dagger} \otimes U_{C}^{\dagger}\right) U_{F}: \mathcal{H}_{R} \otimes \mathcal{H}_{C} \rightarrow \mathcal{H}_{F} \\
E+F & \mapsto U_{E}^{\prime} U_{E}^{\prime \dagger} \otimes \mathbb{1}_{R}+\mathbb{1}_{L} \otimes U_{F}^{\prime} U_{F}^{\prime \dagger}
\end{aligned}
$$

For purposes below, we will also consider the set $Y=\Gamma_{i} \cup \Gamma_{j}$, which we mention here to note that the edge set of $Y$ is not guaranteed to contain all edges joining the vertices of $Y$, in contrast to the other subgraphs described above.

We further note that we can easily determine the dimension of this new space $\mathcal{H}_{\Gamma}$ corresponding to the singular values of some $\Psi_{\Gamma}$. Looking at the collection of virtual indices comprising $\mathcal{H}_{\text {virt }}^{\Gamma}$, we group together those originating from the same site and symmetrize them, which reduces their collective dimension from $2^{k}$ to $k+1$. Then the domain of the resulting space can be obtained as follows: for each vertex $v \in \Gamma$, let $k_{v}$ be the number of "free" indices, that is, edges of $\Lambda$ which terminate in $v$ 
but which do not belong to the subgraph $\Gamma$. Then we expect

$$
\operatorname{dim} \mathcal{H}_{\Gamma}=\prod_{v \in \Gamma}\left(k_{v}+1\right) .
$$

We can confirm equality on a case-by-case basis when performing the singular value decomposition, and, when doing so, the upper bound allows us to rule out the possibility that positive singular values have been improperly discarded due to being below machine precision.

Having completed these preliminaries, we will lay out the three different ways we use these tensors to extract the number $\eta$ :

I Exact diagonalization: We construct $E^{\prime}$ and $F^{\prime}$ explicitly and diagonalize $E^{\prime}+F^{\prime}$, whose eigenvalues are in $[0,2]$. As apparently-integer eigenvalues will only be given up to machine precision, we confirm the apparent eigenspace of 2 by noting that the 2eigenvectors of $E+F$ should correspond to the image of $\Psi_{Y}$, and compare the apparent degeneracy of 2 with the expected dimension of this image. Then we can obtain the greatest non-integer eigenvalue of $E^{\prime}+F^{\prime}$, which will equal $1+\eta$. We use this method to demonstrate the existence of the gap on the squareoctagon, star, and cross lattices.

II Iterative diagonalization with $\Pi_{Y}^{\prime}$ : When $\mathcal{H}_{L} \otimes \mathcal{H}_{C} \otimes$ $\mathcal{H}_{R}$ is too large for us to construct, and therefore diagonalize, $E^{\prime}+F^{\prime}$, we instead iteratively diagonalize it after "shifting" the eigenspace of 2: We note, again, that the 2-eigenspace of $E+F$ should equal the image of $\Psi_{Y}$, or equivalently, that of $E^{\prime}+F^{\prime}$ will equal the image of $\Psi_{Y}\left(U_{L} \otimes U_{C} \otimes U_{R}\right)$. In particular, the projector $\Pi_{Y}^{\prime}$ will project onto the 2-eigenspace of $E^{\prime}+F^{\prime}$; therefore, as long as $E^{\prime}+F^{\prime}$ has any eigenvalues in $(1,2)$, the greatest eigenvalue of $E^{\prime}+F^{\prime}-\Pi_{Y}^{\prime}$ will be the greatest noninteger eigenvalue of $E^{\prime}+F^{\prime}$, i.e. $1+\eta$. Therefore, we can use Lanczos diagonalization procedures (in particular, the high-precision implementation provided by ARPACK) which select the greatest-magnitude eigenvectors of a Hermitian operator. We use this method to demonstrate the existence of the gap on the singly-decorated square and honeycomb lattices.

III Iterative diagonalization with $\Psi_{Y}^{\prime}$ : When we cannot explicitly construct $\Psi_{Y}^{\prime}$, and therefore cannot obtain $U_{Y}^{\prime}$, we can still apply $\Psi_{Y}^{\prime}$ to vectors in $\mathcal{H}_{L} \otimes \mathcal{H}_{C} \otimes$ $\mathcal{H}_{R}$ : in particular we can construct an operator $\rho_{Y} \equiv$ $\Psi_{Y}^{\prime \dagger} \Psi_{Y}^{\prime}$ in order to "shift" the image of $\Psi_{Y}^{\prime}$ as above. In particular, we may seek the greatest eigenvalues of the following operators:

$$
\begin{aligned}
& O_{1} \equiv-\left(E^{\prime} F^{\prime}+F^{\prime} E^{\prime}\right) \\
& O_{2} \equiv \frac{5}{2}\left(E^{\prime}+F^{\prime}\right)-\left(E^{\prime} F^{\prime}+F^{\prime} E^{\prime}\right)-\varepsilon \rho_{Y}
\end{aligned}
$$

where $\varepsilon$ can be tuned as needed (we will choose a value of 0.1 ). Identifying the eigenvalues of $E^{\prime}+F^{\prime}$,

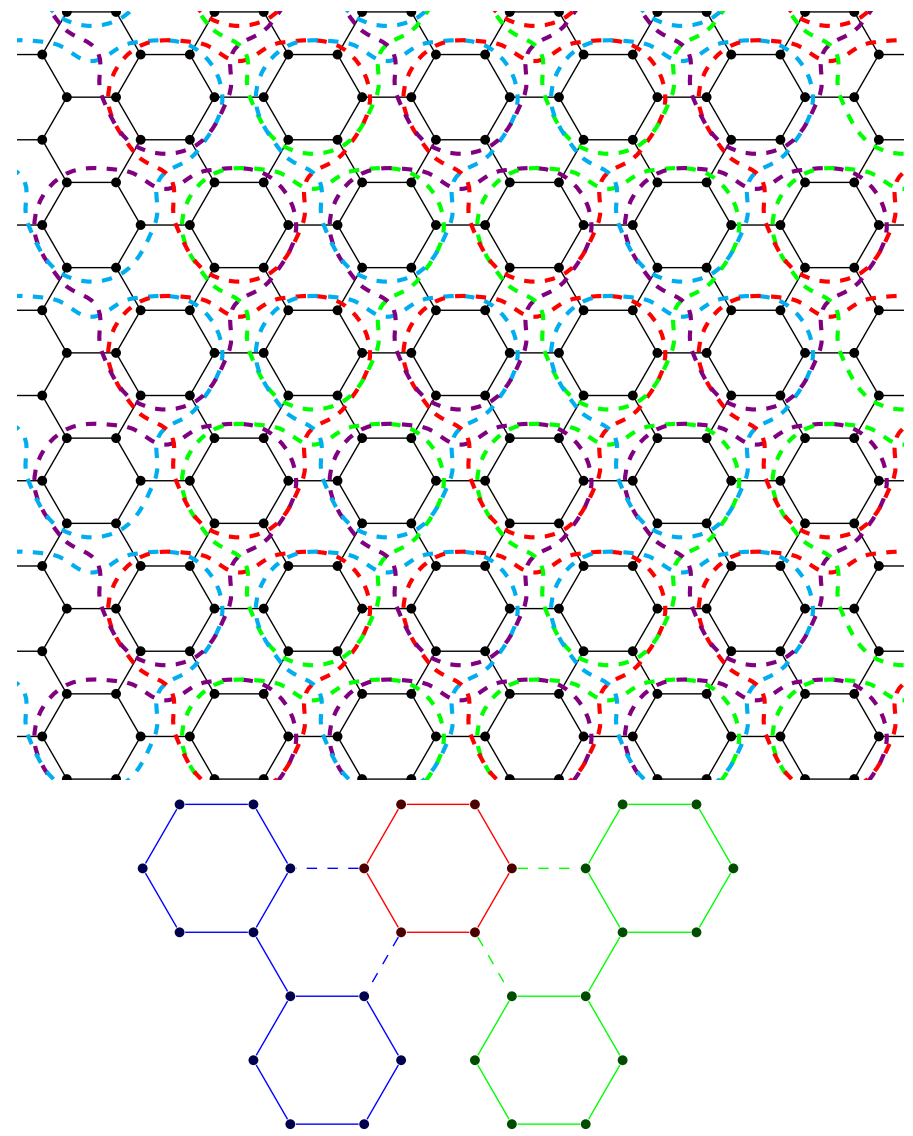

FIG. 1. Above: Enlarged terms of the honeycomb lattice, described below. Below: pairs of adjacent terms, overlapping on hexagonal plaquettes. Blue, green, and red represent the regions on which we apply the "simplifying" isometries $U_{L}$, $U_{R}$, and $U_{C}$, respectively.

as usual, by $1-\alpha$, the eigenvalues of $O_{1}$ are $\alpha(1-\alpha)$, which is maximized by $\alpha=\frac{1}{2}$ : in particular, if $\eta \leq \frac{1}{2}$, then the greatest eigenvalue of $O_{1}$ will be $\eta(1-\eta)$. Meanwhile, for $\alpha \neq-1$, the eigenvalues of $O_{2}$ are $\left(\alpha+\frac{5}{2}\right)(1-\alpha)$, which is maximized by $\alpha=-\frac{3}{4}$. In particular, if there are any $\alpha \in\left(-1,-\frac{1}{2}\right)$, the greatest eigenvalue of $\mathrm{O}_{2}$ will exceed 3 ; meanwhile, if $\eta<\frac{1}{2}$, we ensure by subtracting a multiple of $\rho_{Y}$ that the greatest eigenvalue of $\mathrm{O}_{2}$ is strictly less than 3. We use this method to demonstrate the existence of the AKLT gap on the honeycomb lattice.

\section{RE-PARTITIONING LATTICES}

A summary of values of the key parameter $\eta$, relevant dimensions, and other information is shown in Table 1 


\section{A. The honeycomb lattice}

To find plaquettes we can use to demonstrate the existence of the gap on the honeycomb lattice, we first tripartition the dual lattice; call the resultant sets of plaquettes $\mathcal{A}, \mathcal{B}$, and $\mathcal{C}$. Then, as shown in Fig. 1, we assign for each plaquette $p \in \mathcal{A}$ a subgraph $\Gamma_{p}$ consisting of $p$ and the three neighboring plaquettes belonging to $\mathcal{B}$. Then the overlapping subgraphs consist of nearest neighbors of the triangular lattice whose vertices are the elements of $\mathcal{A}$; in particular, each term overlaps with 6 other terms, so we must find $\eta<\frac{1}{6}$. As shown, the overlapping subgraph $C$ will be a hexagon, whereas the outside subgraphs $L$ and $R$ consist of nonintersecting hexagonal plaquettes joined by a single edge. These allow us to reduce the dimension of the space which $E^{\prime}+F^{\prime}$ acts on to

$$
\begin{aligned}
\operatorname{dim} \mathcal{H}_{C} & =2^{6} \\
\operatorname{dim} \mathcal{H}_{L}=\operatorname{dim} \mathcal{H}_{R} & =2^{10} \\
\operatorname{dim}\left(\mathcal{H}_{L} \otimes \mathcal{H}_{C} \otimes \mathcal{H}_{R}\right) & =2^{26}
\end{aligned}
$$

Then we determine that $\eta=0.1445124916<\frac{1}{6}$.

\section{B. The $[4.8 .8]$ square-octagon lattice}

The new terms we consider correspond to an edge dividing a given pair of octogonal plaquettes and the two square plaquettes it connects. Here each such subgraph overlaps with six others; uniquely among the lattices we are considering, the overlaps between these subgraphs have two different forms. We categorize these terms $\left(\Gamma_{i}\right.$ and $\Gamma_{j}$ ) using the edges connecting the square plaquettes, in that they can be either collinear (Fig. 2 $\mathrm{b}$, say $i \| j$ ) or perpendicular (Fig. 2k, say $i \perp j$ ). We must then modify (9) and (8) to read

$$
\begin{aligned}
\left\{\tilde{H}_{i}, \tilde{H}_{j}\right\} & \geq-\eta_{\|}\left(\tilde{H}_{i}+\tilde{H}_{j}\right), i \| j \\
& \geq-\eta_{\perp}\left(\tilde{H}_{i}+\tilde{H}_{j}\right), i \perp j \\
\tilde{H}^{2} & \geq\left(1-2 \eta_{\|}-4 \eta_{\perp}\right) \tilde{H} .
\end{aligned}
$$

In particular, we need to demonstrate $4 \eta_{\perp}+2 \eta_{\|}<1$. We determine that $\eta_{\|}=0.1061446858$ and $\eta_{\perp}=$ 0.1589663310 .

\section{The [3.12.12] "star" lattice}

Similarly to the square-octagon lattice, in considering the "star" lattice we select subgraphs consisting of the edge dividing a pair of dodecagonal plaquettes and the two triangular plaquettes it connects. Now each such subgraph overlaps with four others, and we find that $\eta=$ $0.1110430220<\frac{1}{4}$.

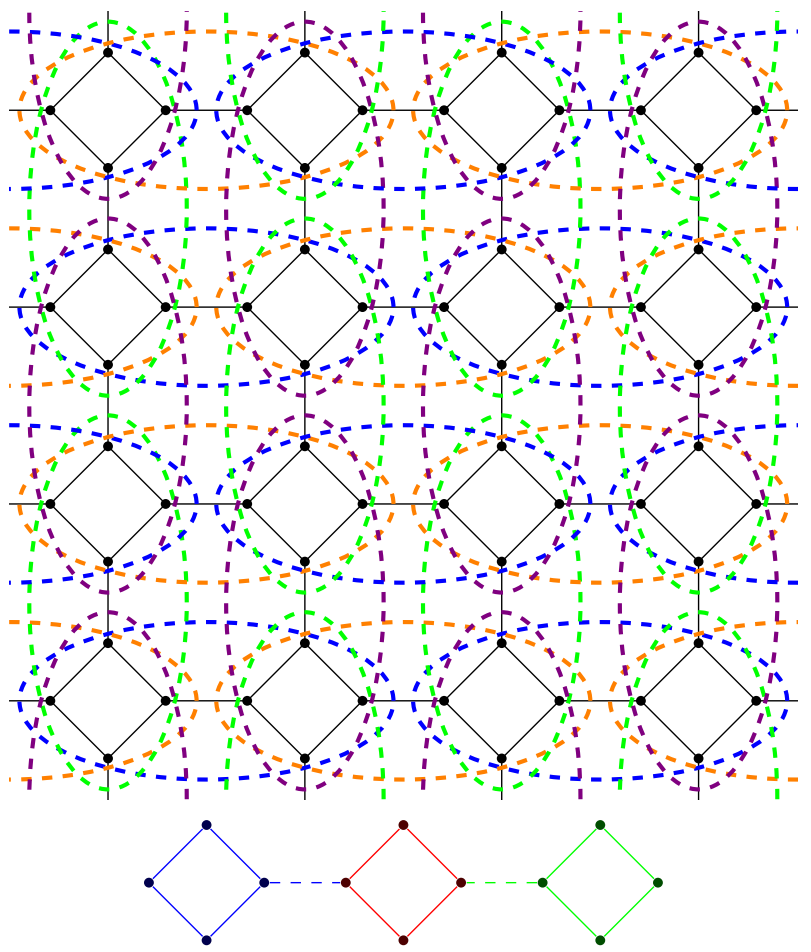

(b)

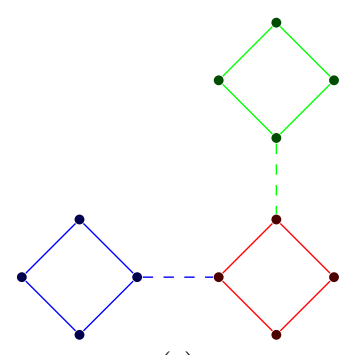

(c)

FIG. 2. Above: Enlarged terms of the square-octagon lattice, corresponding to neighboring pairs of square plaquettes. Below: overlapping terms for which the two octagon-octagon edges are (b) collinear or (c) perpendicular.

\section{The $[4.6 .12]$ "cross" lattice}

In considering the "cross" lattice we select subgraphs corresponding to hexagonal plaquettes, together with the three adjoining square plaquettes. In this case each such subgraph overlaps with three others, one for each square plaquette. We find that $\eta=0.1997384500<\frac{1}{3}$.

\section{E. The honeycomb lattice with $n=1$ decoration}

We now consider the honeycomb lattice with one decoration per edge. We break rotational symmetry and consider each "horizontal" edge of the original honeycomb lattice, connecting vertices $v$ and $w$, together with the decorations on each of the edges "above" $v$ and $w$ and 


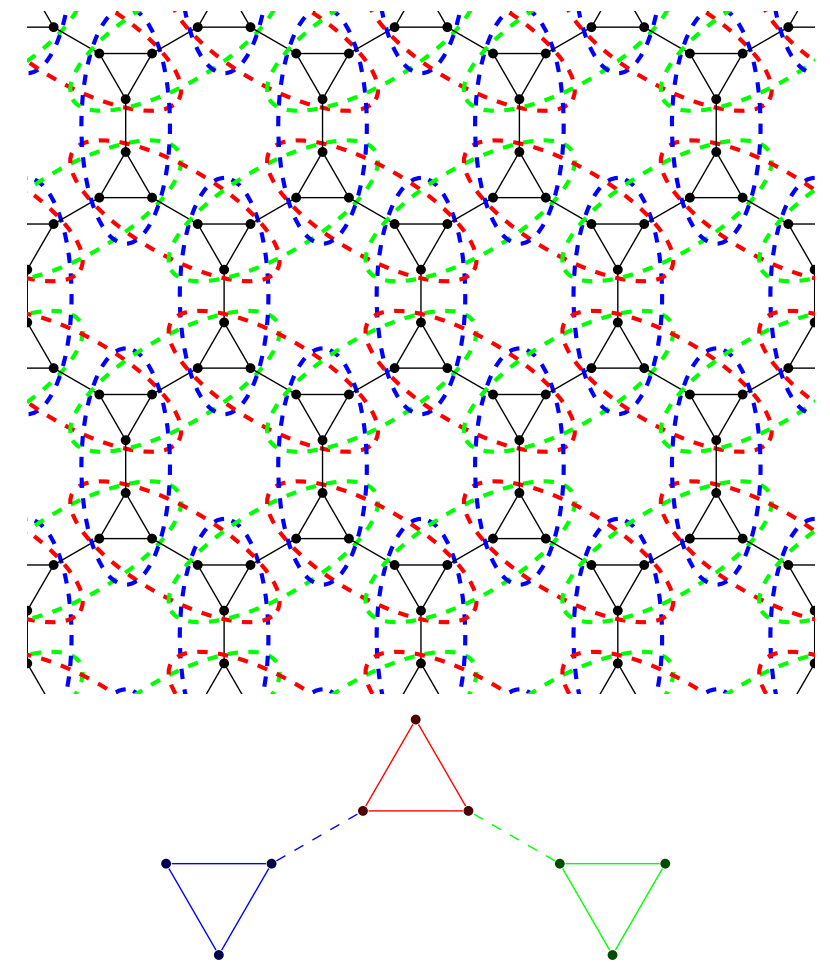

FIG. 3. Above: Enlarged terms of the star lattice, corresponding to neighboring pairs of triangular plaquettes. Below: pairs of adjacent terms, overlapping on triangular plaquettes.

the full edge "below" each of $v$ and $w$, producing the "H" shapes shown in Fig. 5. Then each subgraph overlaps with four others, at each of the four segments (half-edges) at its boundaries. We find that $\eta=0.1530329085<\frac{1}{4}$.

In combination with the results of [13] and [14], this means we can claim conclusively that the AKLT Hamiltonian on the decorated honeycomb lattice is gapped for all $n>0$.

\section{F. The square lattice with $n=1$ decoration}

We conclude by considering the square lattice with one decoration per edge. We first bipartition the dual lattice into sublattices $\mathcal{A}$ and $\mathcal{B}$, and then further bipartition $\mathcal{A}$ into $\mathcal{A}_{1}$ and $\mathcal{A}_{\in}$. The subgraphs we will consider will be of two types, represented in blue and red above in Fig. 6. rotated relative to one another by $\frac{\pi}{2}$. The former consists of a plaquette $p \in \mathcal{A}_{1}$ together with the two decorations adjoining each of the upper-right and lower-left corners of $p$. The latter consists of a plaquette $q \in \mathcal{A}_{2}$ together with the two decorations adjoining each of the upper-left and lower-right corners of $q$. Each of these subgraphs then have four 3 -vertex intersections with other subgraphs, at each of the four corners of the original plaquette. We find that $\eta=0.2203543174<\frac{1}{4}$.

In combination with the results of [14], this means we can claim conclusively that the AKLT Hamiltonian on

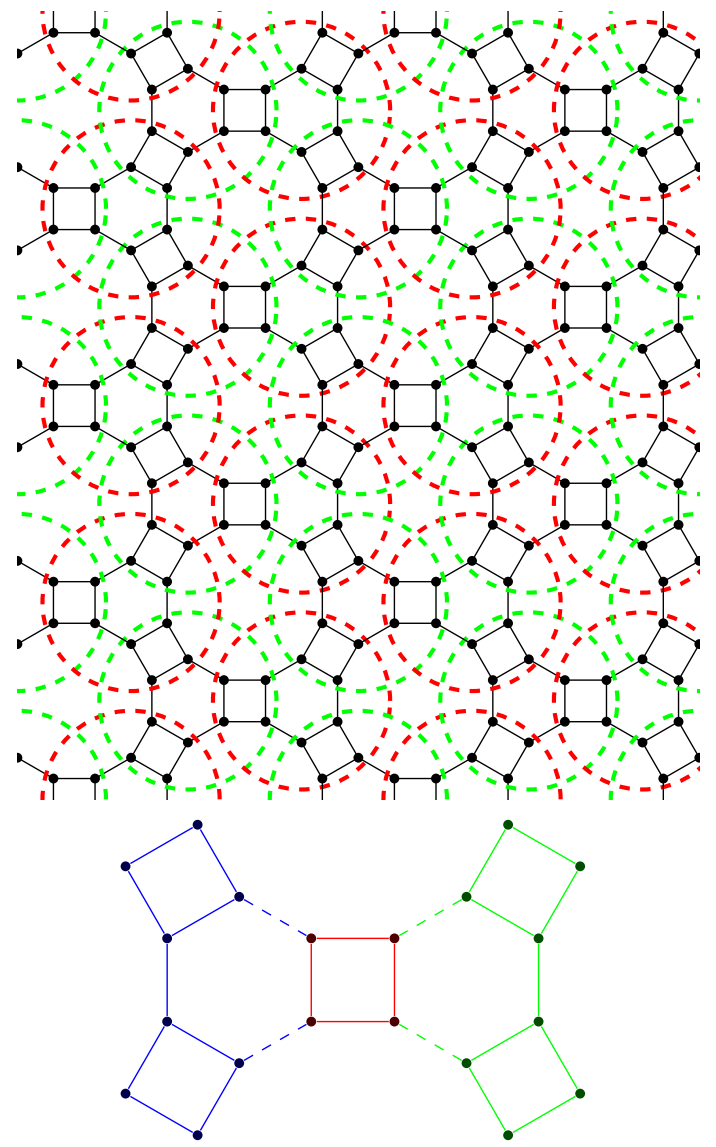

FIG. 4. Above: Enlarged terms of the cross lattice, corresponding to hexagonal plaquettes and the adjoining square plaquettes. Below: pairs of adjacent terms, overlapping on square plaquettes.

the decorated square lattice is gapped for all $n>0$.

\section{COMPLETING THE BOUND ON THE GAP FOR THE HONEYCOMB LATTICE}

In order to produce a bound on the gap of the honeycomb-lattice AKLT lattice, we must bound the original AKLT Hamiltonian relative to the altered Hamiltonian whose gap we have bounded directly, by finding a bound $\gamma_{0}$ as in (3). Here $n_{e}$ will be 3 for each edge belonging to one of the plaquettes in dual sublattice $\mathcal{B}$ and 1 otherwise. Since the total physical dimension supported on one of the chosen subgraphs, $4^{18}$, is far above our capacities, we instead bound $\gamma_{0}$ using intermediate partitions of the subgraph $\Gamma$. To make such an estimate we will need to project out degrees of freedom much as we have already done; but here we will use the following construction:

- For a graph $\Gamma$, whose degrees of freedom factorize according to the vertices of $\Gamma$ as $\mathcal{H}=\bigotimes_{v \in \Gamma} \mathcal{H}_{v}$,

- Suppose that we have a frustration-free Hamilto- 


\begin{tabular}{l|l|l|l|c|c|c|c|c|c} 
& Method & $\eta$ & $\tilde{z}$ & $\operatorname{rank} E$ & $\operatorname{rank} E \wedge F$ & $\operatorname{dim} \mathcal{H}_{L}$ & $\operatorname{dim} \mathcal{H}_{C}$ & $\operatorname{dim} \mathcal{H}_{R}$ & $D_{\text {tot }}$ \\
\hline \hline Square-octagon & I & 0.1061446858 & 2 & $2^{6}$ & $2^{8}$ & $2^{4}$ & $2^{4}$ & $2^{4}$ & $2^{12}$ \\
& I & 0.1589663310 & 4 & & & & & & \\
\hline Star & I & 0.1110430220 & 4 & $2^{4}$ & $2^{5}$ & $2^{3}$ & $2^{3}$ & $2^{3}$ & $2^{9}$ \\
\hline Cross & I & 0.1997384500 & 3 & $2^{6}$ & $2^{8}$ & $2^{6}$ & $2^{4}$ & $2^{6}$ & $2^{16}$ \\
\hline Honeycomb & III & 0.1445124916 & 6 & $2^{12}$ & $2^{18}$ & $2^{10}$ & $2^{6}$ & $2^{10}$ & $2^{26}$ \\
\hline Honeycomb, $n=1$ & II & 0.1530329085 & 4 & $2^{2} 3^{2}$ & $2^{3} 3^{3}$ & $2^{3} 3$ & 6 & $2^{3} 3^{2}$ & $2^{7} 3^{4}$ \\
\hline Square, $n=1$ & II & 0.2203543174 & 4 & $2^{4} 3^{2}$ & $2^{6} 3^{3}$ & $2^{4} 3^{2}$ & $2^{3} 3$ & $2^{6} 3$ & $2^{13} 3^{4}$
\end{tabular}

TABLE I. For each of the configurations considered, the extracted value of $\eta$; the number of intersections $\tilde{z}$ of the type that this particular $\eta$ applies to; the rank of the projectors on the graphs $\Gamma_{i}$ and $\Gamma_{j}, \Gamma_{i} \cup \Gamma_{j}, L=\Gamma_{i} \backslash \Gamma_{j}, C=\Gamma_{i} \cap \Gamma_{j}$, and $R=\Gamma_{j} \backslash \Gamma_{i}$; and the total dimension $D_{\text {tot }} \equiv \operatorname{dim}\left(\mathcal{H}_{L} \otimes \mathcal{H}_{C} \otimes \mathcal{H}_{R}\right)$ of the space on which the operators $E^{\prime}+F^{\prime}$ act.

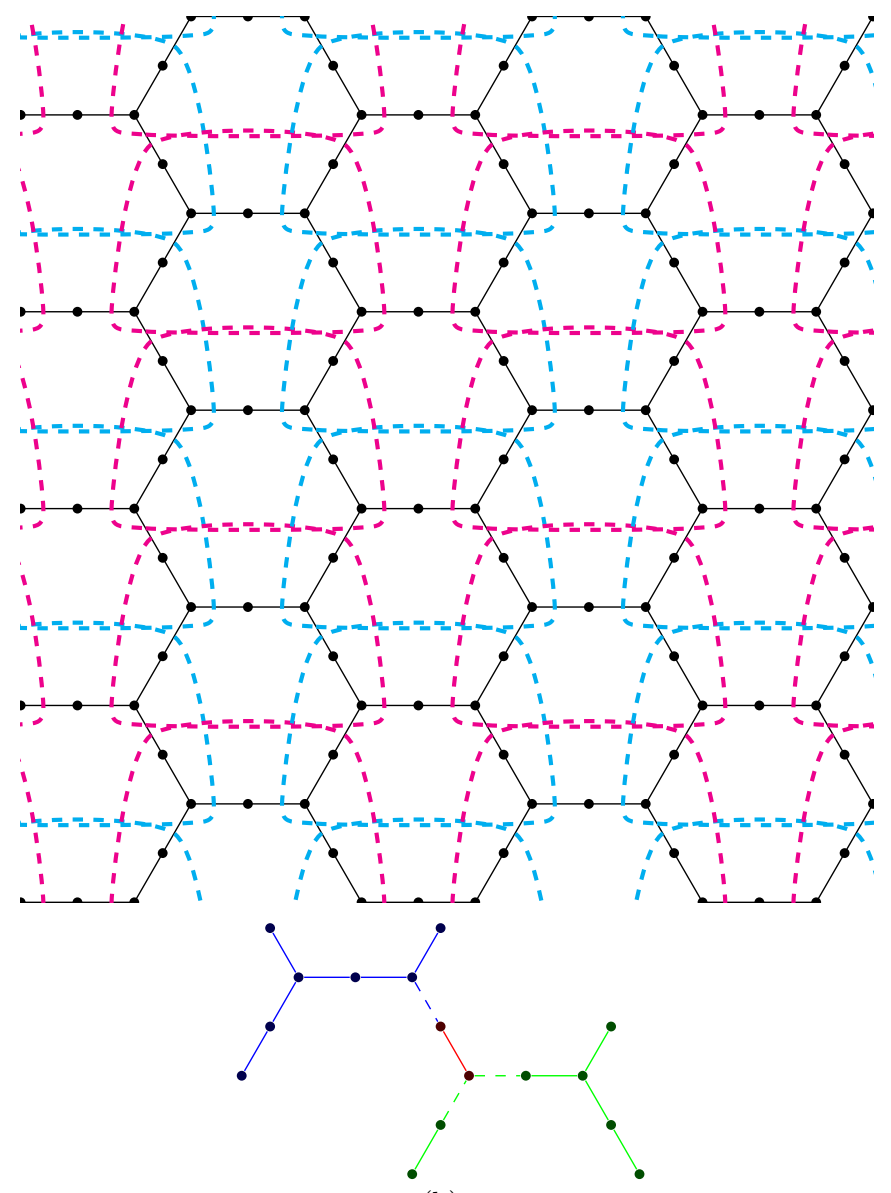

(b)

FIG. 5. Enlarged terms of the once-decorated honeycomb lattice

nian defined as $H_{0}=\sum_{i} c_{i} P_{i}\left(c_{i}>0\right)$, for a collection of projectors $P_{i}$ supported on subgraphs $g_{i} \subset \Gamma$ (which cover $\Gamma$ ), with $\mathbb{1}-\tilde{H}_{0}$ the projector onto the ground space of $H_{0}$

- Consider a collection of isometries $U_{v}: \mathcal{H}_{v} \rightarrow \mathcal{H}_{v}^{\prime}$, corresponding to projectors $\Pi_{v}=U_{v} U_{v}^{\dagger}$, such that,

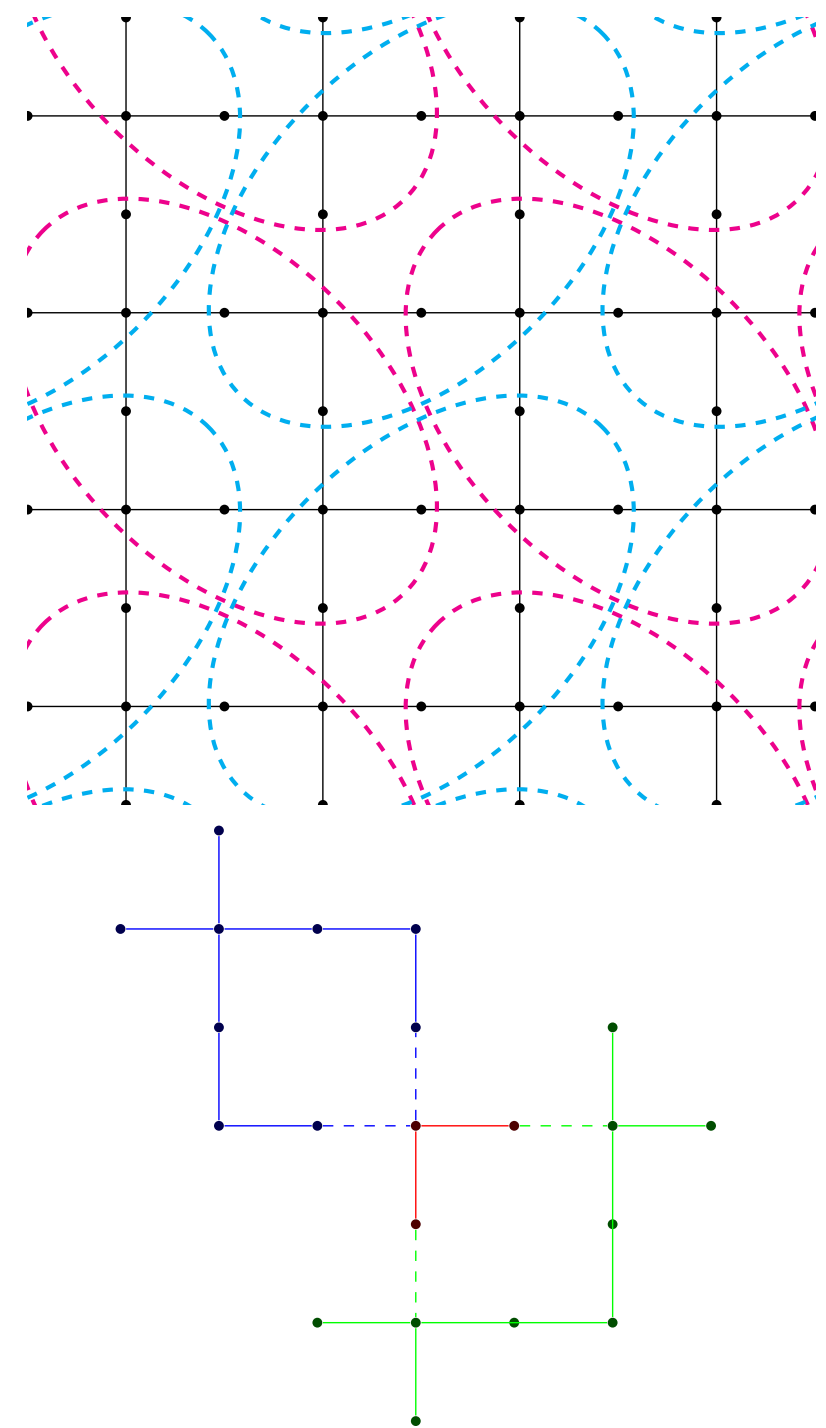

(b)

FIG. 6. Enlarged terms of the once-decorated square lattice 
for all $i$ such that $v \in g_{i}$, then $\Pi_{v}\left(\mathbb{1}-P_{i}\right)=\mathbb{1}-P i$ (noting that, in the cases of interest, this will be an immediate consequence of the above Proposition)

- Let $H_{1}=\mathbb{P} H_{0} \mathbb{P}^{\dagger}$ and $\tilde{H}_{1}=\mathbb{P} \tilde{H}_{0} \mathbb{P}^{\dagger}$, where $\mathbb{P}=$ $\bigotimes_{v \in \Gamma} U_{v}^{\dagger}$. Then

Proposition 2 If $H_{1} \geq \gamma_{1} \tilde{H}_{1}$, then for

$$
\gamma_{0}=\min \left(\gamma_{1}, \min _{i} c_{i}\right)
$$

$H_{0} \geq \gamma_{0} \tilde{H}_{0}$.

Note that, by construction, each "simplifying" projector $\Pi_{v}$ commutes with each of the projectors $P_{i}$ that comprise the Hamiltonian. Therefore, each $\Pi_{v}$ commutes with $H_{0}$ as well as with each other. Thus, we can mutually diagonalize these operators: for an eigenvector $\psi$ of $H_{0}$, with eigenvalue $\lambda$ we can assume that $\psi$ is an eigenvector of each $\Pi_{v}$ as well. If there is a $v$ such that $\Pi_{v} \psi=0$, then, for $g_{i} \ni v,\left(\mathbb{1}-P_{i}\right) \Pi_{v} \psi=0$ implies $P_{i} \psi=\psi$. In particular,

$$
\lambda=\left\langle\psi\left|H_{0}\right| \psi\right\rangle \geq c_{i} \geq \gamma_{0} .
$$

Now suppose that $\Pi_{v} \psi=\psi$ for all $v \in \Gamma$ : in particular, $\mathbb{P}^{\dagger} \mathbb{P} \psi=\psi$. Then $\mathbb{P} \psi \neq 0$ is also an eigenvector of $H_{1}$ with eigenvalue $\lambda$. In particular, if $\lambda \neq 0$ then $\lambda \geq \gamma_{1}$.

We first consider plaquette projectors. We can exactly diagonalize $H_{p, 0}$, the sum over the edges of the plaquette of the terms of the original Hamiltonian; in doing so we determine that $H_{p, 0} \geq \gamma_{p} H_{p}$ for $\gamma_{p}=0.3130520508$, with $\mathbb{1}-H_{p}$ projecting out the ground space of $H_{p, 0}$. Then we can bound

$H_{0}=\frac{1}{3} \sum_{\mathcal{B} \ni e \in \Gamma} H^{(e)}+\sum_{\mathcal{B} \not \supset e \in \Gamma} H^{(e)} \leq \frac{\gamma_{p}}{3} \sum_{\mathcal{B} \ni p \subset \Gamma} H_{p}+\sum_{\mathcal{B} \ngtr e \in \Gamma} H^{(e)}$.

We now consider pairs of plaquettes, as in the subgraphs $L$ and $R$ pictured in Fig. 1, comprised of plaquettes $p$ and $q$ joined by an edge $e$. Now

$$
H_{0}^{\prime}=\frac{\gamma^{[p]}}{6} H_{p}+\frac{\gamma^{[p]}}{6} H_{q}+H^{(e)} .
$$

We will apply the above Proposition to these three terms, applied to a compressed graph $\Gamma$ consisting of each of the two vertices of $e$, the five remaining vertices of $p$ considered as one, and the five remaining vertices of $q$ considered as one. Maximally projecting down the physical spaces of these five-vertex groups, we determine a bound for $H_{0}^{\prime}$ compared with a combined two-plaquette projector $H_{p-q}$,

$$
\gamma^{[2 p]} \leq \min \left(\gamma_{1}^{[2 p]}, \frac{\gamma^{[p]}}{6}\right)=0.02571076873
$$

Thus we can write, for the three $\mathcal{B}$-plaquettes $\{p, q, r\}$,

$$
H_{0} \geq \gamma^{[2 p]}\left(H_{p-q}+H_{q-r}+H_{r-p}\right) \equiv \gamma^{[2 p]} H_{0}^{\prime \prime} .
$$

Now we apply the above proposition again, to a threevertex graph whose vertices are the plaquettes $p, q$, and $r$, and obtain

$$
H_{0}^{\prime \prime} \geq \gamma^{[4 p]} \tilde{H},
$$

for $\gamma^{[4 p]}=0.7784203312$. Then we can write the overall bound on the AKLT Hamiltonian as

$$
\gamma^{[2 p]} \gamma^{[4 p]}(1-6 \eta)=0.002660333395 \text {. }
$$

\section{NOTE ON PRECISION AND ACCURACY}

In general, we have relied on ARPACK methods when exact diagonalization has been available; these generally afford us machine precision, which we confirm by affirming $(E+F) \psi=(1 \pm \eta) \psi$ to within less than $10^{-13}$. It is for this reason that we report 10 digits of precision on our (admittedly somewhat loose) bounds.

Due to time constraints, however, we have not employed full (machine) precision when performing the second diagonalization step of Method III for the honeycomb lattice, that is, finding the greatest eigenvalue of $\mathrm{O}_{2}$ as defined by (14). (We note, for the sake of completeness, that we apply the LM routine to this $\mathrm{O}_{2}$, shifting it by a large constant in order to ensure that the operator remains positive.) Having extracted the greatest eigenvalue of $O_{2}$ with tol parameter $10^{-5}$, we confirm that, for $\lambda$ the eigenvalue and $\psi$ the corresponding eigenvector,

$$
\begin{aligned}
|(E+F) \psi-2 \psi| & +|(E F+F E) \psi-2 \psi| \\
& +|\varepsilon \rho \psi-(3-\lambda) \psi|<10^{-4} .
\end{aligned}
$$

In particular, with $\lambda=2.97451085252$, we can claim an error of no more than $10^{-4}$, which keeps us well within the $\lambda<3$ that we need to confirm that $E+F$ has no eigenvalues within $\left(\frac{3}{2}, 2\right)$.

\section{CONCLUDING REMARKS}

We have closed the open cases in previous works on the spectral gap in AKLT models in decorated honeycomb and square lattices. All these decorated models have nonzero spectral gap, regardless of the number of decorations $n>0$. More relevantly, we have proved the nonzero gap of the original AKLT model on the honeycomb lattice, as well as those on three other degree-3 Archimedean lattices. The lower bound on the spectral gap for the honeycomb case is 0.002550333395 . During the completion of this manuscript, we became aware of a recent preprint [12], in which the gap of the AKLT model on the honeycomb lattice is established, via a combination of analytics and numerical DMRG methods. The spectral gap in spin-2 AKLT models on the square lattice and other degree- 4 lattices should be possible to attack using our method. If the relevant effective matrix $E^{\prime}+F^{\prime}$ to diagonalize is larger than the exact diagonalization 
method or Lanczos, then one may need to resort to other numerical methods. We further note that our method should apply in general to models constructed using the so-called projected-entangled-pair-state (PEPS) formalism, where the ground-space structure can be expressed in terms of exact tensor networks and the parent Hamil- tonian is usually expressed in terms of projectors [25].

\section{ACKNOWLEDGMENTS}

This work was partially supported by the National Science Foundation under grant No. PHY 1915165.
[1] X. G. Wen, Int. J. Mod. Phys. B4, 239 (1990)

[2] Z.-C. Gu and X.-G. Wen, Phys. Rev. B 80, 155131 (2009)

[3] F. Pollmann, E. Berg, A. M. Turner, and M. Oshikawa, Physical Review B 85 (2012).

[4] E. Lieb, T. Schultz, and D. Mattis, Annals of Physics 16, 407 (1961).

[5] M. B. Hastings, Phys. Rev. B 69, 104431 (2004).

[6] M. Oshikawa, Physical Review Letters 84, 1535-1538 (2000)

[7] F. D. M. Haldane, Phys. Rev. Lett. 50, 1153 (1983).

[8] M. B. Hastings and T. Koma, Communications in Mathematical Physics 265, 781 (2006)

[9] I. Affleck, T. Kennedy, E. H. Lieb, and H. Tasaki, Communications in Mathematical Physics 115, 477 (1988)

[10] I. Affleck, T. Kennedy, E. H. Lieb, and H. Tasaki, Phys. Rev. Lett. 59, 799 (1987).

[11] T. Kennedy, E. H. Lieb, and H. Tasaki, Journal of Statistical Physics 53, 383 (1988)

[12] M. Lemm, A. W. Sandvik, and L. Wang, arXiv e-prints (2019), arXiv:1910.11810 [quant-ph].

[13] H. Abdul-Rahman, M. Lemm, A. Lucia, B. Nachtergaele, and A. Young, arXiv e-prints (2019), arXiv:1901.09297 [math-ph].

[14] N. Pomata and T.-C. Wei, Phys. Rev. B 100, 094429 (2019).
[15] T.-C. Wei, I. Affleck, and R. Raussendorf, Phys. Rev. Lett. 106, 070501 (2011)

[16] A. Miyake, Annals of Physics 326, 1656-1671 (2011)

[17] T.-C. Wei, Phys. Rev. A 88, 062307 (2013).

[18] T.-C. Wei, P. Haghnegahdar, and R. Raussendorf, Phys. Rev. A 90, 042333 (2014).

[19] T.-C. Wei and R. Raussendorf, Phys. Rev. A 92, 012310 (2015).

[20] A. Garcia-Saez, V. Murg, and T.-C. Wei, Phys. Rev. B 88, 245118 (2013).

[21] L. Vanderstraeten, M. Mariën, F. Verstraete, and J. Haegeman, Phys. Rev. B 92, 201111 (2015).

[22] D. Poilblanc, N. Schuch, and J. I. Cirac, Phys. Rev. B 88, 144414 (2013)

[23] M. Lemm, A. Sandvik, and S. Yang, "The aklt model on a hexagonal chain is gapped," (2019), arXiv:1904.01043 [quant-ph],

[24] M. Fannes, B. Nachtergaele, and R. F. Werner, Communications in Mathematical Physics 144, 443 (1992)

[25] D. Perez-Garcia, F. Verstraete, M. M. Wolf, and J. I. Cirac, Quantum Info. Comput. 8, 650 (2008) 